\title{
DOMINATED SPLITTINGS FOR FLOWS WITH SINGULARITIES
}

\author{
VITOR ARAUJO, ALEXANDER ARBIETO AND LUCIANA SALGADO
}

\begin{abstract}
We obtain sufficient conditions for an invariant splitting over a compact invariant subset of a $C^{1}$ flow $X_{t}$ to be dominated. In particular, we reduce the requirements to obtain sectional hyperbolicity and hyperbolicity.
\end{abstract}

\section{INTRODUCTION}

The theory of hyperbolic dynamical systems is one of the main paradigm in dynamics. Developed in the 1960s and 1970s after the work of Smale, Sinai, Ruelle, Bowen [27, 26, 9, 10], among many others, this theory deals with compact invariant sets $\Lambda$ for diffeomorphisms and flows of closed finite-dimensional manifolds having a hyperbolic splitting of the tangent space. That is, if $X$ is a vector field and $X_{t}$ is the generated flow then we say that an invariant and compact set $\Lambda$ is hyperbolic if there exists a continuous splitting of the tangent bundle over $\Lambda, T_{\Lambda} M=E^{s} \oplus E^{X} \oplus E^{u}$, where $E^{X}$ is the direction of the vector field, the subbundles are invariant under the derivative $D X_{t}$ of the flow $X_{t}$

$$
D X_{t} \cdot E_{x}^{*}=E_{X_{t}(x)}^{*}, \quad x \in \Lambda, \quad t \in \mathbb{R}, \quad *=s, X, u ;
$$

$E^{s}$ is uniformly contracted by $D X_{t}$ and $E^{u}$ is uniformly expanded: there are $K, \lambda>0$ so that

$$
\left\|\left.D X_{t}\right|_{E_{x}^{s}}\right\| \leq K e^{-\lambda t}, \quad\left\|\left.D X_{-t}\right|_{E_{x}^{u}}\right\| \leq K e^{-\lambda t}, \quad x \in \Lambda, \quad t \in \mathbb{R} .
$$

Very strong properties can be deduced from the existence of a such structure; see for instance [13, 25].

Weaker notions of hyperbolicity, like the notions of dominated splitting, partial hyperbolicity, volume hyperbolicity and singular or sectional hyperbolicity (for singular flows) have been proposed to try to enlarge the scope of this theory to classes of systems beyond the uniformly hyperbolic ones; see [5] and [1] for singular or sectional hyperbolicity. However, the existence of dominated splittings is the weaker one.

Many researchers have studied the relations of the existence of dominated splittings with other dynamical phenomena, mostly in the discrete time case, such as robust transitivity, homoclinic tangencies and heteroclinic cycles, and also the possible extension of this notion to endomorphisms; see for instance $[20,33,21,19,5,6,15]$.

However, the notion of dominated splittings deserves attention. Several authors used this notion for the Linear Poincaré flow, see $[11,16,17]$, and this is useful in the absence of singularities, as in [12]. We remark that this flow is defined only in the set of regular points. However, in the singular case, it is a non-trivial question to obtain a dominated splitting for the derivative of the flow, and thus allowing singularities. Indeed, it is difficult to obtain it, from a dominated splitting for the Linear Poincaré Flow. See [14], for an attempt to solve this, in the context of robustly transitive sets, using the extended Linear Poincaré Flow.

Definition 1.1. A dominated splitting over a compact invariant set $\Lambda$ of $X$ is a continuous $D X_{t^{-}}$ invariant splitting $T_{\Lambda} M=E \oplus F$ with $E_{x} \neq\{0\}, F_{x} \neq\{0\}$ for every $x \in \Lambda$ and such that there are positive constants $K, \lambda$ satisfying

$$
\left\|\left.D X_{t}\right|_{E_{x}}\right\| \cdot\left\|\left.D X_{-t}\right|_{F_{X_{t}(x)}}\right\|<K e^{-\lambda t} \text {, for all } x \in \Lambda \text {, and all } t>0 .
$$

Date: July 9, 2018.

2000 Mathematics Subject Classification. Primary: 37D30; Secondary: 37D25.

Key words and phrases. Dominated splitting, partial hyperbolicity, sectional hyperbolicity.

Authors were partially supported by CNPq, PRONEX-Dyn.Syst., FAPERJ and FAPESB.. 
The purpose of this article is to study the existence of dominated splittings for flows with singularities. In one hand, we study this question in the presence of some sectional hyperbolicity, once that this theory was build to understand flows like the Lorenz attractor, which are the prototype of non-hyperbolic dynamics with singularities, but with robust dynamical properties. On the other hand, we present several examples to clarify the role of the condition on the singularities.

This article is organized as follows. In Section 2, we present definitions and auxiliary results needed in the proof of Theorem A, and some examples showing that the domination condition on the singularities is necessary. In Section 3, we present applications of our main result and more examples implying that these results are not valid for diffeomorphisms. In Section 4 Theorem B is proved assuming Theorem A. In Section 5, we prove the Theorem A and Theorem 2.4. Theorem C and Corollary 2 are proved in Section 6 using the previously proved results. Finally, in Section 7 , we present the examples stated in Propositions 1 and 2.

\section{Statements of the Results}

Let $M$ be a connected compact finite $n$-dimensional manifold, $n \geq 3$, with or without boundary. We consider a vector field $X$, such that $X$ is inwardly transverse to the boundary $\partial M$, if $\partial M \neq \emptyset$. The flow generated by $X$ is denoted by $\left\{X_{t}\right\}$.

An invariant set $\Lambda$ for the flow of $X$ is a subset of $M$ which satisfies $X_{t}(\Lambda)=\Lambda$ for all $t \in \mathbb{R}$. The maximal invariant set of the flow is $M(X):=\cap_{t>0} X_{t}(M)$, which is clearly a compact invariant set.

A singularity for the vector field $X$ is a point $\sigma \in M$ such that $X(\sigma)=\overrightarrow{0}$. The set formed by singularities is denoted by $\operatorname{Sing}(X)$. We say that a singularity is hyperbolic if the eigenvalues of the derivative $D X(\sigma)$ of the vector field at the singularity $\sigma$ have nonzero real part.

A compact invariant set $\Lambda$ is said to be partially hyperbolic if it exhibits a dominated splitting $T_{\Lambda} M=E \oplus F$ such that subbundle $E$ is uniformly contracted, i.e. there exists $C>0$ and $\lambda>0$ such that $\left\|\left.D X_{t}\right|_{E_{x}}\right\| \leq C e^{-\lambda}$ for $t \geq 0$. In this case $F$ is the central subbundle of $\Lambda$.

We say that a $D X_{t}$-invariant subbundle $F \subset T_{\Lambda} M$ is a sectionally expanding subbundle if $\operatorname{dim} F_{x} \geq 2$ is constant for $x \in \Lambda$ and there are positive constants $C, \lambda$ such that for every $x \in \Lambda$ and every two-dimensional linear subspace $L_{x} \subset F_{x}$ one has

$$
\left|\operatorname{det}\left(\left.D X_{t}\right|_{L_{x}}\right)\right|>C e^{\lambda t} \text {, for all } t>0 \text {. }
$$

Definition 2.1. [18, Definition 2.7] A sectional-hyperbolic set is a partially hyperbolic set whose singularities are hyperbolic and central subbundle is sectionally expanding.

This is a generalization of hyperbolicity for compact invariant sets with singularities accumulated by regular orbits, since the Lorenz attractor and higher dimensional examples are sectionalhyperbolic $[30,7,18,1]$ and because of the following result whose proof can be found in $[19,5,1]$.

Lemma 2.2 (Hyperbolic Lemma). Every compact invariant subset of a sectional-hyperbolic set without singularities is a hyperbolic set.

The main result of this paper is the following.

Theorem A. Let $\Lambda$ be a compact invariant set of $X$ such that every singularity in this set is hyperbolic. Suppose that there exists a continuous $D X_{t}$-invariant splitting of the tangent bundle of $\Lambda, T_{\Lambda} M=E \oplus F$, where $E$ is uniformly contracted, $F$ is sectionally expanding and for some constants $C, \lambda>0$ we have

$$
\left\|\left.D X_{t}\right|_{E_{\sigma}}\right\| \cdot\left\|\left.D X_{-t}\right|_{F_{\sigma}}\right\|<C e^{-\lambda t} \quad \text { for all } \sigma \in \Lambda \cap \operatorname{Sing}(X) \text { and } t \geq 0 .
$$

Then $T_{\Lambda} M=E \oplus F$ is a dominated splitting.

As a corollary, we obtain sufficient conditions to obtain hyperbolicity in the non-singular case using the hyperbolic lemma.

Corollary 1. Let $\Lambda$ be a compact invariant set without singularities for the vector field $X$. Suppose that there exists a continuous $D X_{t}$-invariant decomposition of the tangent bundle of $\Lambda, T_{\Lambda} M=$ $E \oplus F$, where $E$ contracts and $F$ is sectionally expanding. Then $\Lambda$ is a hyperbolic set. 
We note that in Definition 2.1 domination is required. As a consequence of Theorem A, the domination assumption is only necessary at the singularities, so that we obtain the following equivalent definition of sectional-hyperbolicity.

Definition 2.3. A compact invariant set $\Lambda \subset M$ is a sectional-hyperbolic set for $X$ if all singularities in $\Lambda$ are hyperbolic, there exists a continuous $D X_{t}$-invariant splitting of the tangent bundle on $T_{\Lambda} M=E \oplus F$ with constants $C, \lambda>0$ such that for every $x \in \Lambda$ and every $t>0$ we have

(1) $\left\|\left.D X_{t}\right|_{E_{x}}\right\| \leq C e^{-\lambda t}$

(2) $\left|\operatorname{det} D X_{t}\right|_{L_{x}} \mid>C^{-1} e^{\lambda t}$, for every two-dimensional linear subspace $L_{x} \subset F_{x}$;

(3) $\left\|\left.D X_{t}\right|_{E_{\sigma}}\right\| \cdot\left\|\left.D X_{-t}\right|_{F_{\sigma}}\right\|<C e^{-\lambda t}$ for all $\sigma \in \Lambda \cap \operatorname{Sing}(X)$.

2.1. Hyperbolicity versus sectional-expansion. We present here some motivation for Theorem A. We observe that, for a hyperbolic set, both splittings $\left(E^{s} \oplus E^{X}\right) \oplus E^{u}$ and $E^{s} \oplus\left(E^{X} \oplus E^{u}\right)$ are dominated. Moreover, it is easy to see that a hyperbolic set $\Lambda$ has no singularities accumulated by regular orbits within $\Lambda$. Indeed, any singularity $\sigma$ accumulated by regular orbits on a hyperbolic set $\Lambda$ would be a discontinuity point for the hyperbolic splitting, due to the absence of the flow direction at $\sigma$.

Our next result shows that we cannot have a hyperbolic splitting without the flow direction, unless we restrict ourselves to finitely many singularities, all of them isolated on the nonwandering set.

Theorem 2.4. Let $\Lambda$ be a compact invariant set of $X$. Suppose that there exists a continuous $D X_{t}$-invariant decomposition of the tangent bundle $T_{\Lambda} M=E \oplus F$ over $\Lambda$ and constants $C, \lambda>0$, such that for every $x \in \Lambda$ and all $t>0$

$$
\left\|\left.D X_{t}\right|_{E_{x}}\right\| \leq C e^{-\lambda t} \quad \text { and } \quad\left\|\left.D X_{-t}\right|_{F_{X_{t}(x)}}\right\| \leq C e^{-\lambda t} .
$$

Then $\Lambda$ consists of finitely many hyperbolic singularities.

We cannot replace the assumptions on Theorem 2.4 either by sectional-expansion or by sectionalcontraction, as the following examples show.

Example 1. Consider a Lorenz-like singularity $\sigma$ for a $C^{1}$ flow $\left\{X_{t}\right\}_{t \in \mathbb{R}}$ on a 3-manifold $M$, that is, $\sigma$ is a hyperbolic singularity of saddle-type such that the eigenvalues of $D X(\sigma)$ are real and satisfy $\lambda_{2}<\lambda_{3}<0<-\lambda_{3}<\lambda_{1}$ and $\lambda_{1}+\lambda_{2}>0$.

Let $E_{i}$ be the eigenspace associated to the eigenvalue $\lambda_{i}, i=1,2,3$, and set $E=E_{3}$ and $F=E_{1} \oplus E_{2}$. Then the decomposition is trivially continuous, not dominated ( $F$ admits vectors more sharply contracted than those of $E$ ) but $E$ uniformly contracts lengths of vectors and $F$ uniformly expands area, that is, $F$ is sectionally-expanded.

Example 2. Consider a hyperbolic saddle singularity, $\sigma$, for a $C^{1}$ flow $\left\{X_{t}\right\}_{t \in \mathbb{R}}$ on a 4-manifold $M$ such that the eigenvalues of $D X(\sigma)$ are real and satisfy

$$
\lambda_{2}<\lambda_{3}<0<\lambda_{4}<\lambda_{1}, \quad \lambda_{1}+\lambda_{3}>0 \quad \text { and } \quad \lambda_{2}+\lambda_{4}<0 .
$$

Let $E_{i}$ be the eigenspace associated to the eigenvalue $\lambda_{i}, i=1,2,3,4$, and set $F=E_{2} \oplus E_{4}$ and $E=E_{1} \oplus E_{3}$. Then the decomposition is trivially continuous, not dominated as before, $E$ is uniformly area contracting, since $\left.D X_{-t}\right|_{E}$ expands area for $t>0$; and $F$ is uniformly area expanding. In other words, $F$ is sectionally-expanded and $E$ is sectionally-contracted.

In both examples above we have sectional-expansion and sectional-contraction along the subbundles of a continuous splitting but the splitting is not dominated. These examples involve a trivial invariant set: an equilibrium point. But there are examples with compact invariant sets having singularities accumulated by regular orbits and also with a dense regular orbit; see Subsection 7.

This suggests that Theorem 2.4 might be generalized if we assume domination at the singularities of $\Lambda$ together with sectional-expansion along $F$ and uniform contraction along $E$ over regular orbits. This is precisely the content of Theorem A. 


\section{Applichtions and other examples}

To present the next result characterizing sectional-hyperbolic sets through sectional Lyapunov exponents, we first have to present more definitions.

We say that a probability measure $\mu$ is $X$-invariant if $\mu\left(X_{t}(U)\right)=\mu(U)$, for every measurable subset $U \subset M$ and every $t \in \mathbb{R}$. Given a compact $X$-invariant set $\Lambda$ we say that a subset $Y \subset M$ is a total probability subset of $\Lambda$ if $\mu(Y)=1$ for every $X$-invariant measure $\mu$ supported in $\Lambda$. We note that each singularity $\sigma$ of $\Lambda$ belongs to $Y$ since $\mu=\delta_{\sigma}$ is a $X$-invariant probability measure.

Let $A: E \times \mathbb{R} \rightarrow E$ be a Borel measurable map given by a collection of linear bijections

$$
A_{t}(x): E_{x} \rightarrow E_{X_{t}(x)}, \quad x \in M, t \in \mathbb{R},
$$

where $M$ is the base space (we assume it is a manifold) of the finite dimensional vector bundle $E$, satisfying the cocycle property

$$
A_{0}(x)=I d, \quad A_{t+s}(x)=A_{t}\left(X_{s}(x)\right) \circ A_{s}(x), \quad x \in M, t, s \in \mathbb{R},
$$

with $\left\{X_{t}\right\}_{t \in \mathbb{R}}$ a smooth flow over $M$. We note that for each fixed $t>0$ the map $A_{t}: E \rightarrow E, v_{x} \in$ $E_{x} \mapsto A_{t}(x) \cdot v_{x} \in E_{X_{t}(x)}$ is an automorphism of the vector bundle $E$.

The natural example of a linear multiplicative cocycle over a smooth flow $X_{t}$ on a manifold is the derivative cocycle $A_{t}(x)=D X_{t}(x)$ on the tangent bundle $T M$ of a finite dimensional compact manifold $M$.

A concrete instance of this structure we will use is the derivative cocycle $A_{t}(x)=D X_{t} \mid E_{x}$ restricted to a continuous $D X_{t}$-invariant subbundle $E$ of the tangent bundle $T M$.

According to the Multiplicative Ergodic Theorem of Oseledets [3, 4], since in this setting we have that $\sup _{-1 \leq t \leq 1} \log \left\|A_{t}(x)\right\|$ is a bounded function of $x \in \Lambda$, there exists a subset $R$ of $\Lambda$ with total probability such that for every $x \in R$ there exists a splitting

$$
E_{x}=E_{1}(x) \oplus \cdots \oplus E_{s(x)}(x)
$$

which is $D X_{t}$-invariant and the following limits, known as the Lyapunov exponents at $x$, exist

$$
\lambda_{i}(x)=\lim _{t \rightarrow \pm \infty} \frac{1}{t} \log \left\|D X_{t}(x) \cdot v\right\|,
$$

for every $v \in E_{i}(x) \backslash\{0\}, i=1, \ldots, s(x)$. We order these numbers as $\lambda_{1}(x)<\cdots<\lambda_{s(x)}(x)$. One of these subbundles is given by the flow direction (at non-singular points of the flow) and the corresponding Lyapunov exponent is zero for almost every point.

The functions $s$ and $\lambda_{i}$ are measurable and invariant under the flow, i.e., $s\left(X_{t}(x)\right)=s(x)$ and $\lambda_{i}\left(X_{t}(x)\right)=\lambda_{i}(x)$ for all $x \in R$ and $t \in \mathbb{R}$. The splitting (3.1) also depends measurably on the base point $x \in R$. If $F$ is a measurable subbundle of the tangent bundle then by "the Lyapunov exponents of $E$ " we mean the Lyapunov exponents of the nonzero vectors in $F$.

Given a vector space $E$, we denote by $\wedge^{2} E$ the second exterior power of $E$, defined as follows. For a basis $v_{1}, \ldots, v_{n}$ of $E$, then $\wedge^{2} E$ is generated by $\left\{v_{i} \wedge v_{j}\right\}_{i \neq j}$. Any linear transformation $A: E \rightarrow F$ induces a transformation $\wedge^{2} A: \wedge^{2} E \rightarrow \wedge^{2} F$. Moreover, $v_{i} \wedge v_{j}$ can be viewed as the 2-plane generated by $v_{i}$ and $v_{j}$ if $i \neq j$; see for instance [3] for more information.

In [2], a notion of sectional Lyapunov exponents was defined and a characterization of sectionalhyperbolicity was obtained based on this notion.

Definition 3.1. [2, Definition 2.2] Given a compact invariant subset $\Lambda$ of $X$ with a $D X_{t}$-invariant splitting $T_{\Lambda} M=E \oplus F$, the sectional Lyapunov exponents of $x$ along $F$ are the limits

$$
\lim _{t \rightarrow+\infty} \frac{1}{t} \log \left\|\wedge^{2} D X_{t}(x) \cdot \widetilde{v}\right\|
$$

whenever they exists, where $\widetilde{v} \in \wedge^{2} F_{x}-\{0\}$.

As explained in [2], if $\left\{\lambda_{i}(x)\right\}_{i=1}^{s(x)}$ are the Lyapunov exponents, then the sectional Lyapunov exponents at a point $x \in R$ are $\left\{\lambda_{i}(x)+\lambda_{j}(x)\right\}_{1 \leq i<j \leq s(x)}$ and they represent the asymptotic sectional-expansion in the $F$ direction.

As an application of Theorem A, we have the following result, which is an extension of the main result in [2] assuming continuity of the splitting, instead of a dominated splitting. 
Theorem B. Let $\Lambda$ be a compact invariant set for a $X$ such that every singularity $\sigma \in \Lambda$ is hyperbolic. Suppose that there is a continuous $D X_{t}$-invariant splitting $T_{\Lambda} M=E \oplus F$ such that $T_{\sigma} M=E_{\sigma} \oplus F_{\sigma}$ is dominated, for every singularity $\sigma \in \Lambda$.

If the Lyapunov exponents in the $E$ direction are negative and the sectional Lyapunov exponents in the $F$ direction are positive on a set of total probability within $\Lambda$, then the splitting is dominated and $\Lambda$ is a sectional hyperbolic set.

We now apply the main result to the setting of weakly dissipative three-dimensional flows.

We recall that an open subset $U$ is a trapping region if $\overline{X_{t}(U)} \subset U, t>0$ and a compact invariant subset $\Lambda$ is attracting if it is the maximal invariant subset $\Lambda(U)=\overline{\cap_{t \geq 0} X_{t}(U)}$ inside the trapping region $U$. We say that a compact invariant subset $\Lambda$ for the flow of the vector field $X$ is weakly dissipative if $\operatorname{div}(X)(x) \leq 0$ for all $x \in \Lambda$, that is, the flow near $\Lambda$ infinitesimally does not expands volume.

We also say that a hyperbolic singularity $\sigma$ of $X$ is $C^{1}$-linearizable if there exists a diffeomorphism $h: V_{\sigma} \rightarrow B$ from an open neighborhood of $\sigma \in M$ to an open neighborhood $B$ of the origin in $\mathbb{R}^{3}$ such that $h\left(X_{t}(x)\right)=e^{t A} \cdot h(x), X_{s}(x) \in V_{\sigma}$ for $|s| \leq t$ and $A=D X(\sigma)$. For this it is enough that the spectrum of $D X(\sigma)$ satisfies a finite number of non-resonance conditions; see e.g. [28]. Hence this is a $C^{r}$-generic condition for all sufficiently big $r \geq 1$.

Theorem C. Let $X$ be a $C^{1}$ vector field on a three-dimensional manifold $M$ admitting a trapping region $U$ whose singularities (if any) are hyperbolic. Let us assume that the compact invariant subset $\Lambda=\Lambda(U)$ is weakly dissipative and endowed with a one-dimensional continuous field $F$ of asymptotically backward contracting directions, that is, $x \in \Lambda \mapsto F_{x}$ is continuous and for each $x \in \Lambda, F_{x}$ is a one-dimensional subspace of $T_{x} M$, and also

$$
\liminf _{t \rightarrow+\infty} \frac{1}{t} \log \left\|D X_{-t} \mid F_{x}\right\|<0 \quad \text { for all } \quad x \in \Lambda \text {. }
$$

If at each $\sigma \in U$ there exists a complementary $D X_{t}$-invariant direction $E_{\sigma}$ such that $E_{\sigma} \oplus F_{\sigma}=$ $T_{\sigma} M$ is a dominated splitting, then $\Lambda$ is a hyperbolic set (in particular, $\Lambda$ has no singularities).

Since we use the attracting and dominated splitting assumption only to prove the non-existence of singularities in $\Lambda$, as a consequence of the proof we obtain the following.

Corollary 2. Let $X$ be a $C^{1}$ vector field on a three-dimensional manifold $M$ admitting compact invariant subset $\Lambda$, without singularities, which is weakly dissipative and endowed with a onedimensional continuous field $F$ of asymptotically backward contracting directions.

Then $\Lambda$ is a hyperbolic set.

These results are extremely weak versions of the following conjecture of Viana, presented in [31].

Conjecture 1. If an attracting set $\Lambda(U)$ of smooth map/flow has a non-zero Lyapunov exponent at Lebesgue almost every point of its isolating neighborhood $U$, that is

$$
\limsup _{t \rightarrow+\infty} \frac{1}{t} \log \left\|D X_{t}\right\|>0 \quad \text { for Lebesgue almost every } \quad x \in U
$$

then $\Lambda(U)$ has a physical measure: there exists an invariant probability measure $\mu$ supported in $\Lambda(U)$ such that for all continuous functions $\varphi: U \rightarrow \mathbb{R}$

$$
\lim _{t \rightarrow+\infty} \frac{1}{t} \int_{0}^{t} \varphi\left(X_{t}(x)\right) d t=\int \varphi d \mu \quad \text { for Lebesgue almost every } \quad x \in U .
$$

Indeed, if $U$ is a trapping region and $\Lambda=\Lambda(U)$ satisfies the assumptions of Theorem $\mathrm{C}$, then $\Lambda$ is a hyperbolic attracting set, for which it is well-known that (3.3) is true and there exists some physical measure; see e.g. $[10,9,5]$.

We now provide several examples which clarify the role of the assumption of domination at the singularities in Theorem A and the relations between dominated splittings for diffeomorphisms and flows.

Proposition 1. There exist the following examples of vector fields and flows. 
(1) A vector field with an invariant and compact set containing singularities accumulated by regular orbits inside the set, with a non-dominated and continuous splitting of the tangent space, but satisfying uniform contraction and sectional-expansion.

(2) A vector field with an invariant and compact set with a continuous and invariant splitting $E \oplus F$ such that $E$ is uniformly contracted, $F$ is area expanding but it is not dominated.

(3) A suspension flow whose base map has a dominated splitting but the flow does not admit any dominated splitting.

Item (3) above is based on a diffeomorphism described in [8] and similar to another suggested by Pujals in [5, Example B.12].

An important remark is that our results are not valid for diffeomorphisms, as the following result shows.

Proposition 2. There exists a transitive hyperbolic set for a diffeomorphism with a non-dominated splitting which is sectionally-expanding and sectionally-contracting.

\section{Proof of Theorem B}

We follows the lines of [2]. The following proposition, whose proof can be found in [2], is the main auxiliary result in the proof.

We fix a compact $X_{t}$-invariant subset $\Lambda$. We say that a family of functions $\left\{f_{t}: \Lambda \rightarrow \mathbb{R}\right\}_{t \in \mathbb{R}}$ is sub-additive if for every $x \in M$ and $t, s \in \mathbb{R}$ we have that $f_{t+s}(x) \leq f_{s}(x)+f_{t}\left(X_{s}(x)\right)$. The Subadittive Ergodic Theorem (see e.g. [32]) shows that the function $\bar{f}(x)=\liminf _{t \rightarrow+\infty} \frac{f_{t}(x)}{t}$ coincides with $\tilde{f}(x)=\lim _{t \rightarrow+\infty} \frac{1}{t} f_{t}(x)$ in a set of total probability in $\Lambda$.

Proposition 3. Let $\left\{t \mapsto f_{t}: \Lambda \rightarrow \mathbb{R}\right\}_{t \in \mathbb{R}}$ be a continuous family of continuous function which is subadditive and suppose that $\bar{f}(x)<0$ in a set of total probability. Then there exist constants $C>0$ and $\lambda<0$ such that for every $x \in \Lambda$ and every $t>0$ we have $\exp \left(f_{t}(x)\right) \leq C \exp \left(\frac{\lambda t}{2}\right)$.

Proof. See [2, Proposition 3.4].

Remark 1. In [2] only $\Lambda=M(X)$ was considered to conclude that $\left\{X_{t}\right\}_{t \in \mathbb{R}}$ is a sectional-Anosov flow. However all statements are valid for a compact invariant subset of $M$.

Proof of Theorem $B$. Define the following families of continuous functions on $\Lambda$

$$
\phi_{t}(x)=\log \left\|\left.D X_{t}\right|_{E_{x}}\right\| \quad \text { and } \quad \psi_{t}(x)=\log \left\|\left.\wedge^{2} D X_{-t}\right|_{F_{X_{t}(x)}}\right\|, \quad(x, t) \in \Lambda \times \mathbb{R} .
$$

Both families $\phi_{t}, \psi_{t}: \Lambda \rightarrow \mathbb{R}$ are easily seen to be subadditive. From Proposition 3 applied to $\phi_{t}(x)$ and the hypothesis on the Lyapunov exponents, there are constants $C>0$ and $\gamma<0$ such that $\exp \left(\phi_{t}(x)\right)=\left\|\left.D X_{t}\right|_{E_{x}}\right\| \leq C \exp (\gamma t)$ for all $t>0$ showing that $E$ is a contractive subbundle.

Analogously, the hypothesis on the sectional Lyapunov exponents and Proposition 3 applied to the function $\psi_{t}(x)$ provides constants $D>0$ and $\eta<0$ for which $\left\|\left.\wedge^{2} D X_{-t}\right|_{F_{X_{t}(x)}}\right\| \leq D e^{\eta t}$, so $F$ is a sectionally expanding subbundle. Now Theorem A ensures that the splitting $E \oplus F$ is a dominated splitting.

\section{Proof of Theorem A And Theorem 2.4}

We need an auxiliary lemma for which the following definition is necessary. Given a pair of subspaces $E_{x}, F_{x}$ of $T_{x} M$ such that $E_{x} \cap F_{x}=\{\overrightarrow{0}\}$, the angle $\angle\left(E_{x}, F_{x}\right)$ between the two subspaces is defined by

$$
\sin \angle\left(E_{x}, F_{x}\right):=\frac{1}{\left\|\pi\left(E_{x}\right)\right\|}, \quad x \in \Lambda,
$$

where $\pi\left(E_{x}\right): E_{x} \oplus F_{x} \rightarrow E_{x}$ is the projection onto $E_{x}$ parallel to $F_{x}$ defined in the vector space $E_{x} \oplus F_{x}$.

We say that an invariant splitting $E \oplus F=T_{\Lambda} M$ of the tangent bundle over a invariant subset $\Lambda$ has angle uniformly bounded away from zero if the dimensions of the fibers $E_{x}, F_{x}$ are constant for all $x$ in $\Lambda$ and there exists $\theta_{0}>0$ such that $\sin \angle\left(E_{x}, F_{x}\right) \geq \theta_{0}$ for each $x$ in $\Lambda$.

The next lemma specifies the subbundle which contains the flow direction. 
Lemma 5.1. Let $\Lambda$ be a compact invariant set for $X$. Given an invariant splitting $E \oplus F$ of $T_{\Lambda} M$ with angle bounded away from zero over $\Lambda$, such that $E$ is uniformly contracted, then the flow direction is contained in the $F$ subbundle, for all $x \in \Lambda$.

Proof. We denote by $\pi\left(E_{x}\right): T_{x} M \rightarrow E_{x}$ the projection on $E_{x}$ parallel to $F_{x}$ at $T_{x} M$, and likewise $\pi\left(F_{x}\right): T_{x} M \rightarrow F_{x}$ is the projection on $F_{x}$ parallel to $E_{x}$. We note that for $x \in \Lambda$

$$
X(x)=\pi\left(E_{x}\right) \cdot X(x)+\pi\left(F_{x}\right) \cdot X(x)
$$

and for $t \in \mathbb{R}$, by linearity of $D X_{t}$ and $D X_{t}$-invariance of the splitting $E \oplus F$

$$
\begin{aligned}
D X_{t} \cdot X(x) & =D X_{t} \cdot \pi\left(E_{x}\right) \cdot X(x)+D X_{t} \cdot \pi\left(F_{x}\right) \cdot X(x) \\
& =\pi\left(E_{X_{t}(x)}\right) \cdot D X_{t} \cdot X(x)+\pi\left(F_{X_{t}(x)}\right) \cdot D X_{t} \cdot X(x)
\end{aligned}
$$

Let $z$ be a limit point of the negative orbit of $x$. That is, we assume without loss of generality since $\Lambda$ is compact, that there is a strictly increasing sequence $t_{n} \rightarrow+\infty$ such that $\lim _{n \rightarrow+\infty} x_{n}:=$ $\lim _{n \rightarrow+\infty} X_{-t_{n}}(x)=z$. Then $z \in \Lambda$ and, if $\pi\left(E_{x}\right) \cdot X(x)$ is not the zero vector, we get

$$
\begin{gathered}
\lim _{n \rightarrow+\infty} D X_{-t_{n}} \cdot X(x)=\lim _{n \rightarrow+\infty} X\left(x_{n}\right)=X(z), \quad \text { but also } \\
\lim _{n \rightarrow+\infty}\left\|D X_{-t_{n}} \cdot \pi\left(E_{x}\right) \cdot X(x)\right\| \geq \lim _{n \rightarrow+\infty} c^{-1} e^{\lambda t_{n}}\left\|\pi\left(E_{x}\right) \cdot X(x)\right\|=+\infty .
\end{gathered}
$$

This is possible only if the angle between $E_{x_{n}}$ and $F_{x_{n}}$ tends to zero when $n \rightarrow+\infty$.

Indeed, using the Riemannian metric on $T_{y} M$, the angle $\alpha(y)=\alpha\left(E_{y}, F_{y}\right)$ between $E_{y}$ and $F_{y}$ is related to the norm of $\pi\left(E_{y}\right)$ as follows: $\left\|\pi\left(E_{y}\right)\right\|=1 / \sin (\alpha(y))$. Therefore

$$
\begin{aligned}
\left\|D X_{-t_{n}} \cdot \pi\left(E_{x}\right) \cdot X(x)\right\| & =\left\|\pi\left(E_{x_{n}}\right) \cdot D X_{-t_{n}} \cdot X(x)\right\| \\
& \leq \frac{1}{\sin \left(\alpha\left(x_{n}\right)\right)} \cdot\left\|D X_{-t_{n}} \cdot X(x)\right\| \\
& =\frac{1}{\sin \left(\alpha\left(x_{n}\right)\right)} \cdot\left\|X\left(x_{n}\right)\right\|
\end{aligned}
$$

for all $n \geq 1$. Hence, if the sequence $\left\|D X_{-t_{n}} \cdot \pi\left(E_{x}\right) \cdot X(x)\right\|$ in the left hand side of (5.1) is unbounded, then $\lim _{n \rightarrow+\infty} \alpha\left(X_{-t_{n}}(x)\right)=0$.

However, since the splitting $E \oplus F$ has angle bounded away from zero over the compact $\Lambda$, we have obtained a contradiction. This contradiction shows that $\pi\left(E_{x}\right) \cdot X(x)$ is always the zero vector and so $X(x) \in F_{x}$ for all $x \in \Lambda$.

The proof of Theorem 2.4 follows from Lemma 5.1.

Proof of Theorem 2.4. We assume, arguing by contradiction, that there exists a point $x \in \Lambda \backslash$ $\operatorname{Sing}(X)$, that is, $x$ is a regular point: $X(x) \neq \overrightarrow{0}$. Hence Lemma 5.1 ensures that $X(x) \in F_{x}$. But the same Lemma 5.1 applied to the reversed flow $X_{-t}$ generated by the field $-X$ on the same invariant set $\Lambda$ shows that $X(x) \in E_{x}$. Thus $X(x) \in E_{x} \cap F_{x}=\{\overrightarrow{0}\}$. This contradiction ensures that $\Lambda \subset \operatorname{Sing}(X)$. But our assumptions on the splitting show that each $\sigma \in \Lambda$ is a hyperbolic singularity: the eigenvalues of $\left.D X\right|_{E_{\sigma}}$ are negative and the eigenvalues of $\left.D X\right|_{F_{\sigma}}$ are positive. It is well-known that hyperbolic singularities $\sigma$ are isolated in the ambient manifold $M$; see e.g. [22]. We conclude that the compact $\Lambda$ is a finite set of hyperbolic singularities.

Before proving Theorem A we need some extra notions and definitions.

We recall that, for a invertible continuous linear operator $L$, the minimal norm (or co-norm) is equal to $m(L):=\left\|L^{-1}\right\|^{-1}$. Hence condition (1.2) in Definition 1.1 is equivalent to

$$
\left\|\left.D X_{t}\right|_{E_{x}}\right\|<K e^{-\lambda t} \cdot m\left(\left.D X_{t}\right|_{F_{x}}\right) \text { for all } x \in \Lambda \text { and for all } t>0 \text {. }
$$

The Multiplicative Ergodic Theorem of Oseledets [3,4] provides the following properties of Lyapunov exponents and Lyapunov subspaces, in addition to the existence of Lyapunov exponents on the total probability subset $R$ of $\Lambda$, as presented in Section 3. For any pair of disjoint subsets 
$I, J \subset\{1, \ldots, s(x)\}$, the angle between the bundles $E_{I}(x)=\bigoplus_{i \in I} E_{i}(x)$ and $E_{J}(x)=\bigoplus_{j \in J} E_{j}(x)$ decreases at most subexponentially along the orbit of $x$, that is

$$
\lim _{t \rightarrow \pm \infty} \frac{1}{t} \log \sin \angle\left(E_{I}\left(X_{t}(x)\right), E_{J}\left(X_{t}(x)\right)\right)=0, \quad x \in R ;
$$

which implies, in particular, that for any pair $i, j \in\{1, \ldots, s(x)\}$ with $i \neq j$ and $v_{i} \in E_{i}(x) \backslash$ $\{\overrightarrow{0}\}, v_{j} \in E_{j}(x) \backslash\{\overrightarrow{0}\}$

$$
\lim _{t \rightarrow \pm \infty} \frac{1}{t} \log \left|\operatorname{det}\left(D X_{t} \mid \operatorname{span}\left\{v_{i}, v_{j}\right\}\right)\right|=\lambda_{i}(x)+\lambda_{j}(x) .
$$

Proof of Theorem A. Let $x \in R \subset \Lambda$ be a regular point of the flow of $X$. From Lemma 5.1 we know that $X(x) \in F_{x}$. Since $F$ is a $D X_{t}$-invariant subbundle, considering the linear multiplicative cocycle $A_{t}(z)=D X_{t} \mid F_{z}$ for $z \in \Lambda, t \in \mathbb{R}$, there exists a splitting $F_{x}=\bigoplus_{j=1}^{s(x)} F_{j}(x)$ of $F_{x}$ into a direct sum of Lyapunov subspaces. One of these subspaces is $E^{X}$ generated by $X(x) \neq \overrightarrow{0}$, which we rename $F_{1}(x)=E_{x}^{X}$ in what follows. We also have the corresponding Lyapunov exponents $\lambda_{j}^{F}(x), j=1, \ldots, s(x)$.

Fixing $i=2, \ldots, s(x)$ and $v \in F_{i}(x) \backslash\{\overrightarrow{0}\}$, we consider $\operatorname{span}\{X(x), v\}$. From the assumption of sectional expansion of area together with the subexponential control on angles at regular points we obtain

$$
0<\lambda \leq \liminf _{t \rightarrow+\infty} \frac{1}{t} \log \left|\operatorname{det}\left(D X_{t} \mid \operatorname{span}\{X(x), v\}\right)\right|=\lambda_{1}^{F}(x)+\lambda_{i}^{F}(x)=\lambda_{i}^{F}(x) .
$$

Hence $\lambda_{i}^{F}(x) \geq \lambda>0$ for all $i=2, \ldots, s(x), x \in R$ and $X(x) \neq \overrightarrow{0}$.

If we consider the cocycle $B_{t}(z)=D X_{t} \mid E_{z}, x \in \Lambda, t \in \mathbb{R}$, then we find the splitting $E_{x}=$ $\bigoplus_{i=1}^{r(x)} E_{j}(x)$ into Lyapunov subspaces and the corresponding Lyapunov exponents $\lambda_{i}^{E}(x), i=$ $1, \ldots, r(x)$. It is easy to see that the assumption of uniform contraction along $E$ implies that $\lambda_{i}^{E}(x) \leq-\lambda<0$ for all $x \in R$ and $1 \leq i \leq r(x)$.

Then $\phi_{t}(x)=\log \frac{\left\|D X_{t} \mid E_{x}\right\|}{m\left(D X_{t} \mid F_{x}\right)}$ is a subadditive family of continuous functions satisfying

$$
\begin{aligned}
\bar{\phi}(x) & =\liminf _{t \rightarrow+\infty} \frac{\phi_{t}(x)}{t} \leq \liminf _{n \rightarrow+\infty} \frac{1}{t} \log \left\|D X_{t} \mid E_{x}\right\|-\limsup _{n \rightarrow+\infty} \frac{1}{t} \log m\left(D X_{t} \mid F_{x}\right) \\
& =\max \left\{\lambda_{i}^{E}(x), 1 \leq i \leq r(x)\right\}-\min \left\{\lambda_{i}^{F}(x), 1 \leq i \leq s(x)\right\} \leq-\lambda-0=-\lambda
\end{aligned}
$$

for all $x \in R$ such that $X(x) \neq \overrightarrow{0}$.

For $x=\sigma$ for some singularity $\sigma \in \Lambda$ we have $\bar{\phi}(\sigma) \leq-\lambda$ as a direct consequence of the assumption of domination at the singularities.

We have shown that $\bar{\phi}(x)<0$ for all $x \in R$. Applying Proposition 3 we see that there exists $C>0$ such that $\exp \phi_{t}(x) \leq C e^{-\lambda t / 2}$ for all $x \in \Lambda$ and $t>0$, which gives us the condition (5.2). This concludes the proof of Theorem A.

The proof of Corollary 1 follows from Theorem A and the Hyperbolic Lemma 2.2.

\section{Proof of Theorem C and Corollary 2}

We start by showing that the one-dimensional asymptotically backward contracting subbundle $F$ over $\Lambda$ is in fact a uniformly expanding subbundle. Indeed, we note that $f_{t}(x)=\log \left\|D X_{-t} \mid F_{x}\right\|$ is a subadditive family of continuous functions satisfying $\bar{f}(x)<0$ for all $x \in \Lambda$. In particular, $\bar{f}(x)<0$ in a set of total probability. Hence by Proposition 3 we obtain $f_{t}(x) \leq C e^{-t \lambda / 2}$ for all $t>0$ and some constants $\lambda, C>0$. (This trivially implies $\left\|D X_{t} \mid F_{X_{t}(x)}\right\| \geq e^{t \lambda / 2} / C, x \in \Lambda, t \geq 0$ and so $\left\|D X_{T} \mid F_{x}\right\|>2$ for all $x \in \Lambda$ and $T \geq 2 \log (2 C) / \lambda$.)

Now we use the assumption that $\Lambda$ is an attracting set whose possible singularities $\sigma$ are hyperbolic, $C^{1}$ linearizable and admit a complementary $D X_{t}$-invariant direction $E_{\sigma}$ such that $E_{\sigma} \oplus F_{\sigma}=T_{\sigma} M$ is a dominated splitting.

Lemma 6.1. The attracting set $\Lambda$ does not contain singularities. 
Proof. By the $C^{1}$-linearization assumption and the Stable/Unstable Manifold Theorems, there exists a corresponding $X_{t}$-invariant (weak- or strong-)unstable one-dimensional manifold $W_{\sigma}^{u}$ contained in $\Lambda$ (since $\Lambda$ is attracting) such that $F_{\sigma}=T_{\sigma} W_{\sigma}^{u}$ and $X_{-t}(z) \underset{t \rightarrow+\infty}{\longrightarrow} \sigma$ for each $z \in W_{\sigma}^{u}$.

Claim 1. For all $z \in W_{\sigma}^{u}$ we have $X(z) \in F_{z}$.

We note that this is trivially true for $z=\sigma$. Let us assume, by contradiction, that $X(z) \notin F_{z}$ for some $z \in W_{\sigma}^{u} \backslash\{\sigma\}$ (and thus for all such $z$ by $D X_{t}$-invariance). By assumption there exists a diffeomorphism $h: V_{\sigma} \rightarrow B$ from an open neighborhood of $\sigma \in M$ to an open neighborhood $B$ of the origin in $\mathbb{R}^{3}$ such that $h\left(X_{t}(x)\right)=e^{t A} \cdot h(x), X_{s}(x) \in V_{\sigma}$ for $|s| \leq t$ and $A=D X(\sigma)$. By a linear change of coordinates we can assume without loss of generality that $D h(\sigma) \cdot F_{\sigma}=\mathbb{R} \times 0^{2}$ and $D h(\sigma) \cdot E_{\sigma}=0 \times \mathbb{R}^{2}$. Let $v: V_{\sigma} \cap \Lambda \rightarrow \mathbb{R}^{3}$ denote the continuous unitary vector field such that $\operatorname{span}\left(v_{x}\right)=D h(x) \cdot F_{x}, x \in V_{\sigma} \cap \Lambda$.

We are assuming that $v_{z}$ has a non-zero component along the $E_{\sigma} \approx 0 \times \mathbb{R}^{2}$ direction for $z \in W_{\sigma}^{u} \backslash\{\sigma\}$. Since $X_{-t}(z) \underset{t \rightarrow+\infty}{\longrightarrow} \sigma$ the domination of the splitting $E_{\sigma} \oplus F_{\sigma}$ ensures that $\angle\left(e^{t A} \cdot v_{z}, E_{\sigma}\right) \stackrel{t \rightarrow-\infty}{\longrightarrow} 0$. However the invariance of $F$ ensures that $F_{X_{-t}(z)} \stackrel{t \rightarrow-\infty}{\longrightarrow} F_{\sigma}$ and since $h$ is a $C^{1}$ conjugation we also have $\angle\left(e^{t A} \cdot v_{z}, F_{\sigma}\right) \stackrel{t \rightarrow-\infty}{\longrightarrow} 0$. This contradiction proves the claim.

Using the claim together with the previous estimate on expansion along $F$, we have that

$$
\infty>\sup _{x \in \Lambda}\|X(x)\| \geq\left\|X\left(X_{k T}(z)\right)\right\|=\left\|D X_{k T} \cdot X(z)\right\| \geq 2^{k}\|X(z)\|, k \geq 1
$$

which is a contradiction and completes the proof of the lemma.

From now we use only the assumptions of Corollary 2, that is, that $\Lambda$ is a weakly dissipative compact invariant subset without singularities having a continuous field of asymptotically backward contracting directions.

In this setting, the Linear Poincaré Flow is well-defined over $\Lambda$. If we denote by $\mathcal{O}_{x}: T_{x} M \rightarrow N_{x}$ the orthogonal projection from $T_{x} M$ to $N_{x}=\left\{v \in T_{x} M:<v, X(x)>=0\right\}$ the orthogonal complement of the field direction at $x \in \Lambda$, then the Linear Poincaré Flow is given by $P_{x}^{t}=$ $\mathcal{O}_{X_{t}(x)} \circ D X_{t}: T_{x} M \rightarrow N_{X_{t}(x)}, x \in \Lambda, t \in \mathbb{R}$.

We note that since the flow direction is invariant, from the splitting $T_{x} M=E_{x}^{X} \oplus N_{X}$ we can write $D X_{t}=\left(\begin{array}{cc}\frac{\left\|X\left(X_{-t}(x)\right)\right\|}{\|X(x)\|} & \star \\ 0 & P^{-t}\end{array}\right)$ and since $\left|\operatorname{det} D X_{-t}\right| \geq 1$ by the weak dissipative assumption, we get $\left|\operatorname{det}\left(P^{-t}\right)\right| \geq m_{0}=\min _{z \in \Lambda} \frac{\|X(x)\|}{\left\|X\left(X_{-t}(x)\right)\right\|}>0$.

Claim 2. The subbundle $\tilde{F}$ of the normal bundle given by $\left\{\tilde{F}_{x}=\mathcal{O}_{x}\left(F_{x}\right)\right\}_{x \in \Lambda}$ is $P^{t}$-invariant and uniformly backward contracting.

Indeed, the continuity of the subbundle $F$ ensures that $\angle\left(F_{x}, X(x)\right)$ is bounded away from zero, and so there exists $\kappa>0$ such that for every $v \in \tilde{F}_{x}$ we have $|\beta| \leq \kappa\|v\|$ such that $\beta X(x)+v=u \in F_{x}$. This ensures that

$$
\begin{aligned}
\left\|P_{x}^{-t} \cdot v\right\| & =\left\|\mathcal{O}_{X_{-t}(x)}\left(D X_{-t} \cdot(u-\beta X(x))\right)\right\| \leq\left\|\mathcal{O}_{X_{-t}(x)}\left(D X_{-t} \cdot u-\beta X\left(X_{-t}(x)\right)\right)\right\| \\
& \leq\left\|D X_{-t} \cdot u\right\| \leq C e^{-t \lambda / 2}\|v+\beta X(x)\| \leq C e^{-t \lambda / 2}(\|v\|+\kappa\|v\|\|X(x)\|) \\
& \leq C\left(1+\kappa \sup _{z \in \Lambda}\|X(z)\|\right) e^{-t \lambda / 2}\|v\|=\tilde{C} e^{-t \lambda / 2}\|v\| .
\end{aligned}
$$

This proves Claim 2.

This is enough to guarantee the existence of a complementary $P^{-t}$-invariant and expanding subbundle through a graph transform technique. To this end, let us consider the one-dimensional subbundle $G_{x}$ of $N_{x}$ orthogonal to $\tilde{F}_{x}$. We can then write $N_{x}=\tilde{F}_{x} \oplus G_{x}$ and so, since $\tilde{F}$ is $P^{t}$-invariant, we get $P_{x}^{-t}=\left(\begin{array}{cc}a_{t}(x) & b_{t}(x) \\ 0 & c_{t}(x)\end{array}\right)$, where $a_{t}(x): \tilde{F}_{x} \rightarrow \tilde{F}_{X_{-t}(x)}, c_{t}(x): G_{x} \rightarrow G_{X_{-t}(x)}$ and $b_{t}(x): G_{x} \rightarrow F_{X_{-t}(x)}$. We know that $\left|a_{t}(x)\right| \leq \tilde{C} e^{-t \lambda / 2}$ and since $0<m_{0} \leq\left|\operatorname{det}\left(P^{-t}\right)\right|=$ $\left|a_{t}(x)\right| \cdot\left|c_{t}(x)\right|$ we obtain $\left|c_{t}(x)\right| \geq m_{0} e^{t \lambda / 2} / \tilde{C}, x \in \Lambda, t>0$. We fix $T>0$ such that $m_{0} e^{T \lambda / 2} / \tilde{C} \geq 2$. 
Let us now consider the family of continuous one-dimensional complementary subspaces of $\tilde{F}_{x}$ inside $N_{x}$ given by the graph of a linear map $L: G_{x} \rightarrow \tilde{F}_{x}$, i.e., we consider the vector space $\mathcal{G}$ of all $\mathcal{L}=\left\{L_{x}: G_{x} \rightarrow \tilde{F}_{x}: x \in \Lambda\right\}$ with the norm $\left\|\mathcal{L}^{1}-\mathcal{L}^{2}\right\|=\sup _{x \in \Lambda}\left\|L_{x}^{1}-L_{x}^{2}\right\|$. We have that $\mathcal{G}$ with this norm is a Banach space since $\Lambda$ is compact.

It is easy to see that the map $P^{-t}$ transforms the graph of $L_{x}$ into the graph of $\hat{L}_{X_{-t}(x)}=$ $\left(P_{x}^{-t} \cdot L_{x}+\pi_{X_{-t}(x)} \cdot P^{-t}\right) \circ\left[\left(I-\pi_{X-t}(x)\right) \cdot P^{-t}\right]^{-1}$, where $\pi_{z}: N_{z} \rightarrow \tilde{F}_{z}$ is the orthogonal projection from $N_{z}$ into $\tilde{F}_{z}$ at $z \in \Lambda$. In this way we get a map $P^{-T}: \mathcal{G} \rightarrow \mathcal{G}$ which we claim is a contraction with respect to the norm given above. Indeed, for any pair $\mathcal{L}^{i}=\left\{L_{x}^{i}\right\}_{x \in \Lambda}, i=1,2$ we get

$$
\begin{aligned}
\left\|\hat{L}_{X_{-T}(x)}^{1}-\hat{L}_{X_{-T}(x)}^{2}\right\| & =\left\|P_{x}^{-t} \cdot\left(L_{x}^{1}-L_{x}^{2}\right) \circ\left[\left(I-\pi_{X_{-t}(x)}\right) \cdot P^{-t}\right]^{-1}\right\| \\
& \leq\left|a_{T}(x)\right| \cdot\left\|L_{x}^{1}-L_{x}^{2}\right\| /\left|c_{t}(x)\right| \leq \frac{1}{2}\left\|L_{x}^{1}-L_{x}^{2}\right\|
\end{aligned}
$$

for each $x \in \Lambda$. We thus have a $P^{-T}$ fixed point $\mathcal{L}^{0}$ defining a $P^{-T}$-invariant subbundle $\tilde{E}=$ $\left\{\tilde{E}_{x}=\left\{\left(u, L_{x}^{0}(u)\right): u \in G_{x}\right\}_{x \in \Lambda}\right.$ of the normal bundle. Since $P^{-T}$ commutes with each $P^{s}$ for all $s \in \mathbb{R}$, we see that $\tilde{E}$ is $P^{s}$-invariant for all $s \in \mathbb{R}$.

Claim 3. The subbundle $E=\tilde{E} \oplus E^{X}$ is $D X_{t}$-invariant.

Indeed, for any $v=u+\beta X(x)$ with $u \in \tilde{E}_{x}, \beta \in \mathbb{R}$, we have $D X_{t} \cdot v=D X_{t} \cdot u+\beta X\left(X_{t}(x)\right)$ and $D X_{t} \cdot u=P_{x}^{t} \cdot u+\gamma X\left(X_{t}(x)\right)$ for some $\gamma \in \mathbb{R}$, thus $D X_{t} \cdot v=P_{x}^{t} \cdot u+(\beta+\gamma) X\left(X_{t}(x)\right)$ with $P_{x}^{t} \cdot u \in \tilde{E}_{X_{t}(x}$, hence $D X_{t} \cdot v \in E_{X_{t}(x)}$ and the claim is proved.

At this point we have a continuous splitting $T_{x} M=E_{x} \oplus F_{x}, x \in \Lambda$. Using this invariant splitting we can write $D X_{-t}=\left(\begin{array}{cc}A_{t}(x) & 0 \\ 0 & B_{t}(x)\end{array}\right)$ and the continuity of the splitting ensures that $\angle\left(E_{x}, F_{x}\right)$ is bounded away from zero. This ensures that $1 \leq\left|\operatorname{det} D X_{-t}\right|=\left|\operatorname{det} A_{t}(x)\right| \cdot\left|B_{t}(x)\right| \cdot \sin \angle\left(E_{x}, F_{x}\right)$ and since $B_{t}(x)=D X_{-t} \mid F_{x}$ we have $\left|B_{t}(x)\right| \leq C e^{-t \lambda / 2}$, hence $\left|\operatorname{det} A_{t}(x)\right|$ grows exponentially fast.

This shows that $\Lambda$ has a continuous invariant splitting $E \oplus F$ with $F$ uniformly contracting and $E$ area expanding for the backward flow. In a three-dimensional manifold this shows that $E$ is sectionally-expanding for $-X$. From Corollary 1 we have that $\Lambda$ is a hyperbolic set for $-X$, hence $\Lambda$ is a hyperbolic set and the proof of Theorem $\mathrm{C}$ and Corollary 2 is complete.

\section{EXAMPLES}

This section is devoted to present some examples.

7.1. Proof of Proposition 1. Here we present examples stated in Proposition 1.

Item (1): Consider a vector field on the plane $\mathbb{R}^{2}$ with a double saddle homoclinic connection which expands volume and multiply this vector field by a contraction along the vertical direction; see Figure 1. For the construction of the planar vector field, see e.g. [23, Chapter 4, Section 9].

The compact invariant set $W$, for the vector field $X$, formed by the pair of homoclinic connections together with the singularity $s$ admits a splitting $E \oplus F$, where $E$ is the vertical direction and $F$ is the plane direction. This splitting is continuous, $E$ is uniformly contracting and $F$ is uniformly area expanding.

Indeed, since we assume that the singularity $s$ expands area, i.e., $\left|\operatorname{det} D X_{t}(x)\right|_{L_{x}} \mid \geq c e^{\lambda t}$ for all $t>0$ and all 2-plane $L_{x} \subset F_{x}$, for some fixed constants $c, \lambda>0$, we can fix a neighborhood $U$ of $s$ such that

$$
\left|\operatorname{det} D X_{t}(x)\right|_{L_{x}} \mid \geq c e^{\lambda t}, \forall 0 \leq t \leq 1, \forall x \in U
$$

and consequently

$$
\left|\operatorname{det} D X_{t}(x)\right|_{L_{x}} \mid \geq c e^{\lambda t}, \forall t>0
$$

whenever the positive orbit of $x$ is contained in $U$. Now we observe that there exists $T>0$ such that for all $x \in W \backslash U$ we have $X_{t}(x) \in U$ for all $t \in \mathbb{R}$ satisfying $|t|>T$. Hence we can choose 


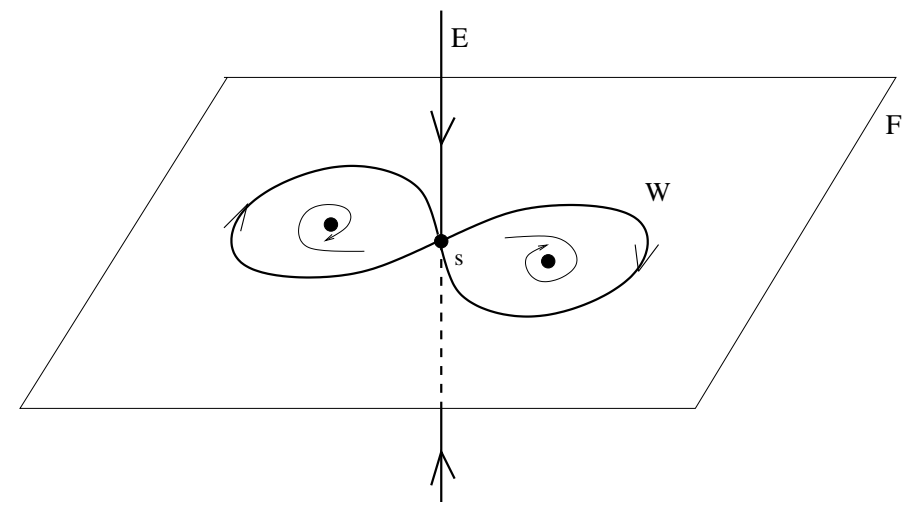

FiguRE 1. A double homoclinic saddle connection expanding area

$k=\inf \left\{c^{-1} e^{-\lambda t}\left|\operatorname{det} D X_{t}(x)\right|_{L_{x}} \mid: x \in W \backslash U, 0 \leq t \leq T\right\}>0$ and write $\left|\operatorname{det} D X_{t}(x)\right|_{L_{x}} \mid \geq k \cdot c e^{\lambda t}$ for $0 \leq t \leq T$; and for $t>T$

$\left.\left.\left|\operatorname{det} D X_{t}(x)\right|_{L_{x}}|=| \operatorname{det} D X_{t-T}\left(X_{T}(x)\right)\right|_{L_{X_{T}(x)}}|\cdot| \operatorname{det} D X_{T}(x)\right|_{L_{x}} \mid \geq c e^{\lambda(t-T)} \cdot k \cdot c e^{\lambda T}=k c^{2} e^{\lambda t}$.

So we can find a constant $\kappa>0$ so that $\left|\operatorname{det} D X_{t}(x)\right|_{L_{x}} \mid \geq \kappa e^{\lambda t}$ for all $x \in W$ and every $t \geq 0$.

To obtain a non-dominated splitting, just choose the contraction rate along $E$ to be weaker than the contraction rate of the singularity $s$.

Example 3. Considering, in the previous Item (1), $\Lambda$ as the union of the saddle s with only one homoclinic connection, we have $T_{\Lambda} M=E \oplus F$ a non-dominated continuous splitting over a compact invariant and isolated set containing a singularity of the flow. Moreover, $\Lambda$ is the $\alpha$-limit set of all points of the plane $\mathbb{R}^{2} \times\{0\}$ in the region bounded by the curve $\Lambda$ except the sink; see [23, Chapter 4, Section 9].

Item (2): Consider the flow known as "Bowen example"; see e.g. [29] and Figure 2. We have

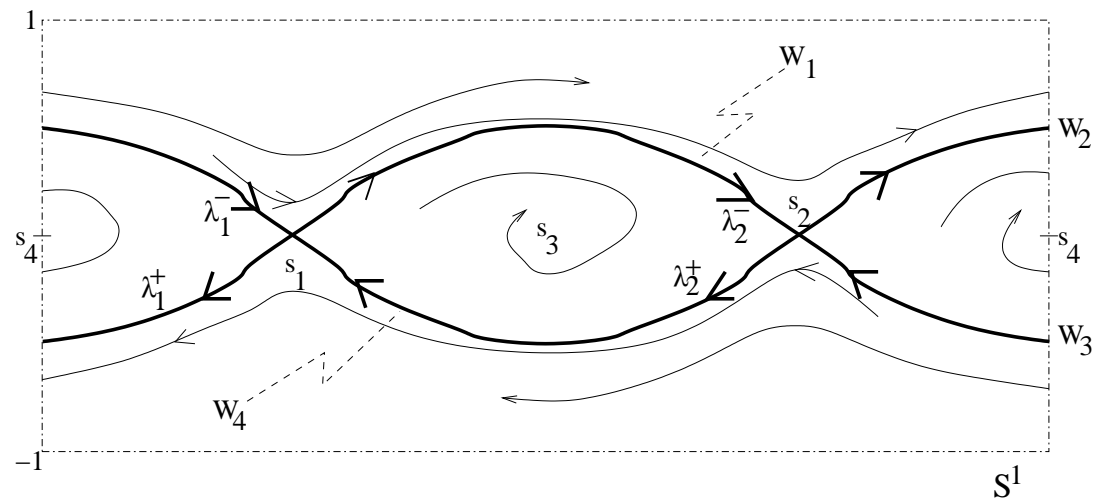

Figure 2. A sketch of Bowen's example flow.

chosen to reverse time with respect to the flow studied in [29] so that the heteroclinic connection $W=\left\{s_{1}, s_{2}\right\} \cup W_{1} \cup W_{2} \cup W_{3} \cup W_{4}$ is now a repeller. The past orbit under this flow $\phi_{t}$ of every point $z \in S^{1} \times[-1,1]=M$ not in $W$ accumulates on either side of the heteroclinic connection, as suggested in the figure, if we impose the condition $\lambda_{1}^{-} \lambda_{2}^{-}<\lambda_{1}^{+} \lambda_{2}^{+}$on the eigenvalues of the saddle fixed points $s_{1}$ and $s_{2}$ (for more specifics on this see [29] and references therein). In this setting, the saddle singularities $s_{1}, s_{2}$ are area expanding: $\left|\operatorname{det} D \phi_{t}\left(s_{i}\right)\right|$ grows exponentially with $t>0$, $i=1,2$. 
We can now embed this system in the 2-sphere putting two sinks at the "north and south poles" of $\mathbb{S}^{2}$; see Figure 3. Let us denote the corresponding vector field on $\mathbb{S}^{2}$ by $X$.

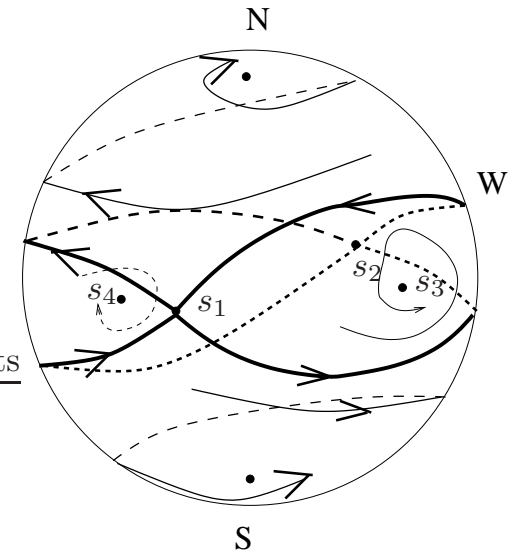

Figure 3. The Bowen flow embedded in a sphere

Let $Y$ be a vector field in $\mathbb{S}^{1}$ corresponding to the "north-south" system: the corresponding flow has only two fixed points $N$ and $S, N$ is a source and $S$ a sink. We can choose the absolute value of the eigenvalues of $Y$ at $N, S$ to be between the absolute value of the eigenvalues of $s_{1}, s_{2}$.

In this way, for the flow $\left\{Z_{t}\right\}_{t \in \mathbb{R}}$ associated to the vector field $X \times Y$ on $\mathbb{S}^{2} \times \mathbb{S}^{1}$, the singularities $\left\{s_{i} \times S\right\}_{i=1,2}$ of the invariant set $\Lambda:=W \times\{S\}$ are hyperbolic saddles, and the splitting $T_{\Lambda}\left(\mathbb{S}^{2} \times\right.$ $\left.\mathbb{S}^{1}\right)=E \oplus F$ given by

$$
E_{(p, S)}=\{0\} \times T_{S} \mathbb{S}^{1} \quad \text { and } \quad F_{(p, S)}=T_{p} \mathbb{S}^{2} \times\{0\} \quad \text { for } \quad p \in W,
$$

is continuous, $D Z_{t}$-invariant, $E$ is uniformly contracted and $F$ is area expanding (by an argument similar to the previous example), but it is not dominated.

Example 3 and items (1) and (2) of Proposition 1 show that Theorem A is false without the dominating condition on the splitting $E \oplus F$ at the set of singularities within $\Lambda$.

Item (3): Now we present a suspension flow whose base map has a dominated splitting but the flow does not admit any dominated splitting.

Let $f: \mathbb{T}^{4} \times \mathbb{T}^{4}$ be the diffeomorphism described in [8] which admits a continuous dominated splitting $E^{c s} \oplus E^{c u}$ on $\mathbb{T}^{4}$, but does not admit any hyperbolic (uniformly contracting or expanding) subbundle. There are hyperbolic fixed points of $f$ satisfying (see Figure 4):

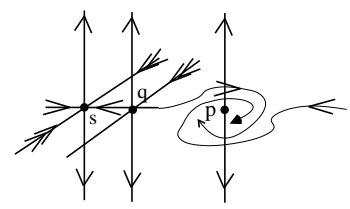

Figure 4. Saddles with real and complex eigenvalues.

- $\operatorname{dim} E^{u}(p)=2=\operatorname{dim} E^{s}(p)$ and there exists no invariant one-dimensional sub-bundle of $E^{u}(p)$

- $\operatorname{dim} E^{u}(\widetilde{p})=2=\operatorname{dim} E^{s}(\widetilde{p})$ and there exists no invariant one-dimensional sub-bundle of $E^{s}(\widetilde{p})$

- $\operatorname{dim} E^{s}(\widetilde{q})=3$ and $\operatorname{dim} E^{u}(q)=3$.

Hence, the suspension flow of $f$ with constant roof function 1 does not admit any dominated splitting. In fact, the natural invariant splitting $E^{c s} \oplus E^{X} \oplus E^{c u}$ is the continuous invariant 
splitting over $\mathbb{T}^{4} \times[0,1]$ with bundles of least dimension, and is not dominated since at the point $p$ the flow direction $E^{X}(p)$ dominates the $E^{c s}(p)=E^{s}(p)$ direction, but at the point $q$ this domination is impossible.

This completes the proof of Proposition 1.

7.2. Proof of Proposition 2. We present first some auxiliary notions.

Let $f: M \rightarrow M$ be a $C^{1}$-diffeomorphism and $\Gamma \subset M$ a compact $f$-invariant set, that is, $f(\Gamma)=\Gamma$. We say that $\Gamma$ is transitive if there exists $x \in \Gamma$ with dense orbit: the closure of $\left\{f^{n}(x): n \geq 1\right\}$ equals $\Gamma$. The invariant set $\Gamma$ is topologically mixing if, for each pair $U, V$ of non-empty open sets of $\Gamma$, there exists $N=N(U, V) \in \mathbb{Z}^{+}$such that $U \cap f^{n}(V) \neq \emptyset$ for all $n>N$.

We say that $\Gamma$ is an attracting set if there exists a neighborhood $U$ of $\Gamma$ in $M$ such that $\cap_{n \geq 0} f^{n}(U)=\Gamma$; in this case we say that $U$ is an isolating neighborhood for $\Gamma$. An attractor is a transitive attracting set and a repeller is an attractor for the inverse diffeomorphism $f^{-1}$. It is well-known that hyperbolic attractors or repellers can be decomposed into finitely many compact subsets which are permuted and each of these pieces is topologically mixing for a power of the original map; see e.g. [25].

Now, we present the example stated in Proposition 2. Consider a hyperbolic attractor (a Plykin attractor, see [24]) $\Gamma$, with an isolating neighborhood $U$, defined on the two-dimensional sphere $\mathbb{S}^{2}$ for a diffeomorphism $f: \mathbb{S}^{2} \rightarrow \mathbb{S}^{2}$, with a splitting $T_{\Gamma} \mathbb{S}^{2}=E \oplus F$ satisfying $\left\|\left.D f\right|_{E}\right\| \leq \lambda_{s}$ and $\left\|\left.D f\right|_{F}\right\| \geq \lambda_{u}$ where $0<\lambda_{s}<1<\lambda_{u}$ and $\lambda_{s} \cdot \lambda_{u}<1$.

Now we consider the following diffeomorphism

$$
g=f_{1} \times f_{2}: M \rightarrow M, \text { with } M=\mathbb{S}^{2} \times \mathbb{S}^{2},
$$

where $f_{1}:=f^{2}$ and $f_{2}:=f^{-1}$. We note that $\Gamma_{1}:=\cap_{n \geq 0} f_{1}^{n}(U)$ is a hyperbolic attractor with respect to $f_{1}$ whose contraction and expansion rates along its hyperbolic splitting $T_{\Gamma_{1}} \mathbb{S}^{2}=E_{1} \oplus F_{1}$ are $\lambda_{s}^{2}, \lambda_{u}^{2}$, respectively. Likewise $\Gamma_{2}:=\cap_{n \leq 0} f_{2}^{n}(U)$ is a hyperbolic repeller with respect to $f_{2}$ whose contraction and expansion rates along its hyperbolic splitting $T_{\Gamma_{2}} \mathbb{S}^{2}=E_{2} \oplus F_{2}$ are $\lambda_{u}^{-1}, \lambda_{s}^{-1}$, respectively. This implies in particular that $D f_{1}$ contracts area near $\Gamma_{1}$, that is, $\lambda_{s}^{2} \cdot \lambda_{u}^{2}<1$ and that $D f_{2}$ expands area near $\Gamma_{2}$, that is, $\left(\lambda_{s} \cdot \lambda_{u}\right)^{-1}>1$. Moreover, the set

$$
\Gamma:=\Gamma_{1} \times \Gamma_{2}
$$

is a hyperbolic set for $g$, whose hyperbolic splitting is given by $\left(E_{1} \times E_{2}\right) \oplus\left(F_{1} \times F_{2}\right)$.

Now we note, on the one hand, that the splitting $T_{\Gamma} M=E \oplus F$ with $E=E_{1}$ and $F=$ $F_{1} \oplus E_{2} \oplus F_{2}$ is not dominated, since the contraction rate along $E_{2}$ is stronger than the contraction rate along $E_{1}$, but $E$ is uniformly contracted and $F$ is uniformly sectionally-expanded, since the Lyapunov exponents $2 \log \lambda_{u},-\log \lambda_{u},-\log \lambda_{s}$ are such that each pair has positive sum.

On the other hand, the decomposition $T_{\Gamma} M=T_{\Gamma_{1}} \mathbb{S}^{2} \times T_{\Gamma_{2}} \mathbb{S}^{2}=E \oplus F$ where $E=E_{1} \oplus F_{1}$ and $F=E_{2} \oplus F_{2}$ is continuous, since each subbundle is continuous; is also $D g$-invariant; but it is not a dominated decomposition. In fact, we have the contraction/expansion rates $0<\lambda_{s}^{2}<1<\lambda_{u}^{2}$ associated to $E$ and the corresponding rates $\lambda_{u}^{-1}, \lambda_{s}^{-1}$ associated to $F$, satisfying the relation $\lambda_{s}^{2}<\lambda_{u}^{-1}<1<\lambda_{s}^{-1}<\lambda_{u}^{2}$ (since $0<\lambda_{s} \cdot \lambda_{u}<1$ and $0<\lambda_{s}<1<\lambda_{u}$ imply that $\lambda_{s}^{2}<\lambda_{u}^{-1}$ and $\left.\lambda_{s}^{-1}<\lambda_{u}^{2}\right)$. Therefore the splitting $E \oplus F$ cannot be dominated. In addition, as noted above, both $\left.D g^{-1}\right|_{E}$ and $\left.D g\right|_{F}$ expand area, so that $E$ is sectionally-contracted and $F$ is secionally-expanded.

Finally, since both $\left.f_{1}\right|_{\Gamma_{1}}$ and $\left.f_{2}\right|_{\Gamma_{2}}$ are topologically mixing, then $\left.g\right|_{\Gamma}$ is transitive; see e.g. [32].

This example shows that there are transitive hyperbolic sets with a non-dominated, although continuous, splitting satisfying the sectional-expansion and uniform contraction or sectional contraction conditions. This completes the proof of Proposition 2.

\section{ACKNOWLEDGMENTS}

This is a part of the PhD thesis of L. Salgado at Instituto de Matemática-Universidade Federal do Rio de Janeiro under a CNPq (Brazil) scholarship and she is now supported by INCTMatCAPES post-doctoral scholarship at IMPA.

The authors thank C. Morales and L. Díaz for many observations that helped improve the statements of the results. 


\section{REFERENCES}

[1] V. Araújo and M. J. Pacifico. Three-dimensional flows, volume 53 of Ergebnisse der Mathematik und ihrer Grenzgebiete. 3. Folge. A Series of Modern Surveys in Mathematics [Results in Mathematics and Related Areas. 3rd Series. A Series of Modern Surveys in Mathematics]. Springer, Heidelberg, 2010. With a foreword by Marcelo Viana.

[2] A. Arbieto. Sectional lyapunov exponents. Proc. of the Amercian Mathematical Society, 138:3171-3178, 2010.

[3] L. Arnold. Random dynamical systems. Springer-Verlag, Berlin, 1998.

[4] L. Barreira and Y. Pesin. Nonuniform hyperbolicity, volume 115 of Encyclopedia of Mathematics and its Applications. Cambridge University Press, Cambridge, 2007. Dynamics of systems with nonzero Lyapunov exponents.

[5] C. Bonatti, L. J. Díaz, and M. Viana. Dynamics beyond uniform hyperbolicity, volume 102 of Encyclopaedia of Mathematical Sciences. Springer-Verlag, Berlin, 2005. A global geometric and probabilistic perspective, Mathematical Physics, III.

[6] C. Bonatti, N. Gourmelon, and T. Vivier. Perturbations of the derivative along periodic orbits. Ergodic Theory Dynam. Systems, 26(5):1307-1337, 2006.

[7] C. Bonatti, A. Pumariño, and M. Viana. Lorenz attractors with arbitrary expanding dimension. C. R. Acad. Sci. Paris Sér. I Math., 325(8):883-888, 1997.

[8] C. Bonatti and M. Viana. SRB measures for partially hyperbolic systems whose central direction is mostly contracting. Israel J. Math., 115:157-193, 2000.

[9] R. Bowen. Equilibrium states and the ergodic theory of Anosov diffeomorphisms, volume 470 of Lect. Notes in Math. Springer Verlag, 1975.

[10] R. Bowen and D. Ruelle. The ergodic theory of Axiom A flows. Invent. Math., 29:181-202, 1975.

[11] C. I. Doering. Persistently transitive vector fields on three-dimensional manifolds. In Procs. on Dynamical Systems and Bifurcation Theory, volume 160, pages 59-89. Pitman, 1987.

[12] S. Gan and L. Wen. Nonsingular star flows satisfy Axiom A and the no-cycle condition. Invent. Math., 164(2):279-315, 2006.

[13] A. Katok and B. Hasselblatt. Introduction to the modern theory of dynamical systems, volume 54 of Encyclopeadia Appl. Math. Cambridge University Press, Cambridge, 1995.

[14] M. Li, S. Gan, and L. Wen. Robustly transitive singular sets via approach of an extended linear Poincaré flow. Discrete Contin. Dyn. Syst., 13(2):239-269, 2005.

[15] C. Liang and G. Liu. Dominated splitting versus small angles. Acta Math. Sin. (Engl. Ser.), 24(7):1163-1174, 2008.

[16] S. T. Liao. On the stability conjecture. Chinese Annals of Math, 1:9-30, 1980.

[17] R. Mañé. An ergodic closing lemma. Annals of Math., 116:503-540, 1982.

[18] R. Metzger and C. Morales. Sectional-hyperbolic systems. Ergodic Theory and Dynamical System, 28:15871597, 2008.

[19] C. A. Morales, M. J. Pacifico, and E. R. Pujals. Robust transitive singular sets for 3-flows are partially hyperbolic attractors or repellers. Ann. of Math. (2), 160(2):375-432, 2004.

[20] K. Moryiasu. The extension of dominated splittings for $c^{1}$-regular maps. Tokyo J. Math., 14(2):425-437, 1991.

[21] S. Newhouse. Cone-fields, domination, and hyperbolicity. In Modern dynamical systems and applications, pages 419-432. Cambridge Univ. Press, Cambridge, 2004.

[22] J. Palis and W. de Melo. Geometric Theory of Dynamical Systems. Springer Verlag, 1982.

[23] L. Perko. Differential equations and dynamical systems, volume 7 of Texts in Applied Mathematics. SpringerVerlag, New York, third edition, 2001.

[24] R. V. Plykin. Sources and currents of A-diffeomorphisms of surfaces. Math USSR Sbornik, 94:243-264, 1974.

[25] C. Robinson. An introduction to dynamical systems: continuous and discrete. Pearson Prentice Hall, Upper Saddle River, NJ, 2004.

[26] Y. Sinai. Markov partitions and C-diffeomorphisms. Func. Anal. and Appl., 2:64-89, 1968.

[27] S. Smale. Differentiable dynamical systems. Bull. Am. Math. Soc., 73:747-817, 1967.

[28] E. Sternberg. On the structure of local homeomorphisms of euclidean n-space - II. Amer. J. Math., 80:623-631, 1958.

[29] F. Takens. Heteroclinic attractors: time averages and moduli of topological conjugacy. Bull. Braz. Math. Soc., 25:107-120, 1995.

[30] W. Tucker. The Lorenz attractor exists. C. R. Acad. Sci. Paris, 328, Série I:1197-1202, 1999.

[31] M. Viana. Dynamics: a probabilistic and geometric perspective. In Proceedings of the International Congress of Mathematicians, Vol. I (Berlin, 1998), number I in Extra Vol., pages 557-578 (electronic), 1998.

[32] P. Walters. An introduction to ergodic theory. Springer Verlag, 1982.

[33] L. Wen. Homoclinic tangencies and dominated splittings. Nonlinearity, 15(5):1445-1469, 2002.

(V.A.) Instituto de Matemática, Universidade Federal da Bahia, Av. Adhemar de Barros, S/N, Ondina, 40170-110 - SALVAdOR-BA-BraziL

E-mail address: vitor.d.araujo@ufba.br 
(A.A.) Universidade Federal do Rio de Janeiro, Instituto de Matemática, P. O. Box 68530, 21945-970 Rio de JANEIRo, BraziL

E-mail address: arbieto@im.ufrj.br

(L.S.) Instituto de Matemática Pura e Aplicada - Estrada Dona Castorina, 110, Jardim Botânico, 22460-320 Rio De Janeiro, Brazil

E-mail address: lsalgado@impa.br 